\title{
Apoptosis coincident with the differentiation of skeletal myoblasts is delayed by caspase 3 inhibition and abrogated by MEK-independent constitutive Ras signaling
}

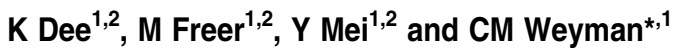 \\ 1 Department of Biology, Geology and Environmental Science, Cleveland State \\ University, Cleveland, $\mathrm{OH} 44115$, USA \\ 2 These authors contributed equally to this work \\ * Corresponding author: CM Weyman, Dept. B.G.E.S., SRC 266, 2399 Euclid \\ Avenue, Cleveland State University, Cleveland, OH 44115, USA. \\ Tel: +1 2166876971 ; Fax: +1 216687 6972; E-mail: c.weyman@csuohio.edu
}

Received 6.4.01; revised 25.6.01; accepted 13.7.01

Edited by DR Green

\begin{abstract}
We demonstrate that during 23A2 skeletal myoblast differentiation, between $30-35 \%$ of the population apoptose. Both differentiation and apoptosis are controlled by the variables of cell density and time and these variables are inversely related. In response to conditions that permit both differentiation and apoptosis of parental 23A2 myoblasts, myoblasts rendered differentiation-defective by constitutive Ras signaling (A2:HRas myoblasts) do not apoptose. This is not merely a consequence of their differentiation-defective phenotype since myoblasts rendered differentiation-defective by expression of E1A (A2:E1A myoblasts) still apoptose. Although signaling through MEK is important to the survival of proliferating parental 23A2 myoblasts, constitutive signaling through MEK is not responsible for the survival of A2:H-Ras myoblasts. Finally, we demonstrate that caspase 3 is activated and that pharmacological inhibition of caspase 3 activity delays apoptosis without affecting differentiation. Abrogating apoptosis without affecting differentiation could be a useful approach to improve the efficacy of myoblast transfer in the treatment of muscular dystrophies.

Cell Death and Differentiation (2002) 9,209-218. DOI: 10.1038/sj/ $\mathrm{cdd} / 4400930$
\end{abstract}

Keywords: apoptosis; differentiation; skeletal myoblast; Ras

Abbreviations: MRFs, muscle regulatory transcription factors; GM, growth medium; DM, differentiation medium; MHC, myosin heavy chain; BME, basal modified Eagle's medium; FBS, fetal bovine serum; TdT, terminal deoxynucleotidyl transferase; PS, phosphatidyl serine; PBS, phosphate-buffered saline

\section{Introduction}

Apoptosis occurs in all tissues and is as critical to normal development and homeostasis as proliferation, migration and differentiation. ${ }^{1,2}$ As testament to the vital importance of apoptosis, inappropriate apoptosis is associated with many pathological conditions. ${ }^{3}$ Like the apoptosis of thymocytes and neurons, ${ }^{1,2}$ the apoptosis of myoblasts is a physiological process during myogenesis ${ }^{4}$ and regeneration and likely serves the critical function of removing excess myoblasts. ${ }^{5}$ During the process of differentiation, apoptosis has been documented in cultures of primary myoblasts ${ }^{6}$ and in established muscle cell lines. ${ }^{7}$ Inappropriate myoblast apoptosis contributes pathologically to the degeneration associated with various muscular dystrophies ${ }^{8,9}$ and spinal muscular atrophies. ${ }^{10,11}$ Furthermore, despite great advances in vector design and protocols which decrease immune responses, ${ }^{12,13}$ myoblast therapy as an approach to the treatment of inherited muscular dystrophies is still severely impaired by the poor survival of transplanted myoblasts. ${ }^{14,15}$

The therapeutic manipulation of myoblasts can be facilitated through a complete molecular understanding of their biology. The very idea that myoblast transfer is a conceptually viable approach to treat myopathies originated over 20 years ago from a fundamental understanding about how multinucleated skeletal muscle is formed. ${ }^{16}$ Since then, a combination of studies in cell lines and mice has delineated the specific roles of a family of muscle regulatory transcription factors (MRFs) composed of MyoD, Myogenin, Myf-5 and MRF4. Much is also known about the signaling pathways regulating the MRFs. ${ }^{17}$ Signaling by appropriate concentrations of IGFs through the PI3-kinase/ Akt pathway ${ }^{18-21}$ is known to be required for differentiation while signaling by FGF-2 or high concentrations of IGF through the Raf/MEK pathway is known to inhibit differentiation. $^{22}$ Despite the wealth of molecular knowledge about skeletal myoblast determination, proliferation, and differentiation, information regarding skeletal myoblast apoptosis is limited to a few reports that during the process of differentiation induced by culture in low serum containing medium, roughly $30 \%$ of myoblasts die via apoptosis. ${ }^{7}$ The ability to escape this apoptosis seems to be linked to cell cycle exit. $^{7,23,24}$

The molecules responsible for the apoptotic process have not been examined in myoblasts but have been extensively studied in other systems. Apoptosis is tightly regulated by a continually emerging plethora of molecular checks and balances designed to control the activation of a family of cysteine, aspartic acid specific proteases (caspases). This complex array of regulatory molecules makes possible the distinct regulation of caspase activation in each system. Caspases naturally exist as procaspases which are themselves activated by limited proteolytic processing after aspartic acid. Procaspases are generally divided into two classes. 'Initiator' caspases like caspases 8 and 9 contain prodomains that permit aggregation in response to an apoptotic signal. ${ }^{25,26}$ Aggregation permits allosteric activation ${ }^{27}$ as well as activation as a conse- 
quence of trans- or self-processing. Activated initiator caspases then activate the 'executioner' caspases which include caspases 3, 6 and $7 .{ }^{28}$ Caspase activation via this cascade of limited proteolysis then results in the limited proteolysis of a discrete set of critical cellular proteins. ${ }^{25,26}$

To expand our extremely limited knowledge about the regulation of apoptosis that occurs during the differentiation of skeletal myoblasts, we carefully examined the conditions that result in the apoptosis of 23A2 skeletal myoblasts. We have documented that differentiation and apoptosis depend on the variables of cell density and time and that these variables are inversely related. We have determined that, under conditions that permit both differentiation and apoptosis of parental 23A2 myoblasts, constitutive signaling by Ras inhibits both their differentiation ${ }^{29}$ and their apoptosis while signaling by $\mathrm{E} 1 \mathrm{~A}$ inhibits only their differentiation. Thus, the inhibition of differentiation can be separated from the inhibition of apoptosis. We used PD098059, a selective pharmacological inhibitor of MEK, to show that while MEK signaling does play a role in the survival of $23 \mathrm{~A} 2$ myoblasts under growth conditions, the Ras-induced inhibition of apoptosis under differentiationinducing conditions is MEK independent. To investigate further the possible separation of signals required for differentiation from those signals required for apoptosis, we determined that pharmacological inhibition of caspase 3 signaling delays apoptosis without affecting differentiation. Inhibiting apoptosis without affecting differentiation could eventually be a viable approach to preventing the deterioration associated with many muscular dystrophies and could also improve the therapeutic efficacy of myoblast transfer.

\section{Results}

\section{Differentiation and apoptosis are cell density and time dependent}

Apoptosis has been documented in $\mathrm{C} 2 \mathrm{C} 12$ myoblasts induced to differentiate by switching cultures from high serum growth medium to low serum differentiation medium. ${ }^{7}$ We too switched cultures from high serum growth medium (GM) to differentiation medium without serum (DM) to document the induction of both differentiation and apoptosis in 23A2 myoblasts and also extended these studies to document differentiation and apoptosis as a consequence of extended culture in GM. We chose to also perform these studies in GM as this is possibly more representative of the in vivo process. Furthermore, we analyzed the effect of varying cell density and time on differentiation and apoptosis for both approaches.

To monitor differentiation, we used myosin heavy chain $(\mathrm{MHC})$ as our marker and documented its expression by both Western analysis (Figure $1 \mathrm{~A}$ ) of lysates and by immunostaining of cell cultures (Figure 1B, C). The Western analysis is quantitative with respect to the amount of $\mathrm{MHC}$ that is expressed while the immunostain is quantitative with respect to the percentage of cells that have initiated $\mathrm{MHC}$ expression. By plating cells at the same density and monitoring $\mathrm{MHC}$ expression over time in either $\mathrm{DM}$ or $\mathrm{GM}$, we can document that differentiation is time dependent (Figure 1A, B). By plating cells at different densities and monitoring $\mathrm{MHC}$ expression after the same time in either DM or GM, we can also show that differentiation is density dependent (Figure 1C). It is clear from both analyses that these variables of time and density are inversely related. It is also clear that differentiation occurs faster when cells are switched from GM to DM than under extended culture in GM, for example, compare $24 \mathrm{~h}$ in DM to $24 \mathrm{~h}$ in GM (Figure 1A, B). The lag time between the differentiation that occurs under extended culture in GM and the differentiation that occurs when cells are switched from GM to DM is roughly $24 \mathrm{~h}$ (Figure $1 \mathrm{C}$ ).

To monitor apoptosis, we used DNA fragmentation as our marker and documented it by both immunostaining of cell cultures (Figure 2) and ELISA of cytosolic lysates (Figure 3). The immunostain is quantitative with respect to the percentage of cells that have initiated apoptosis and
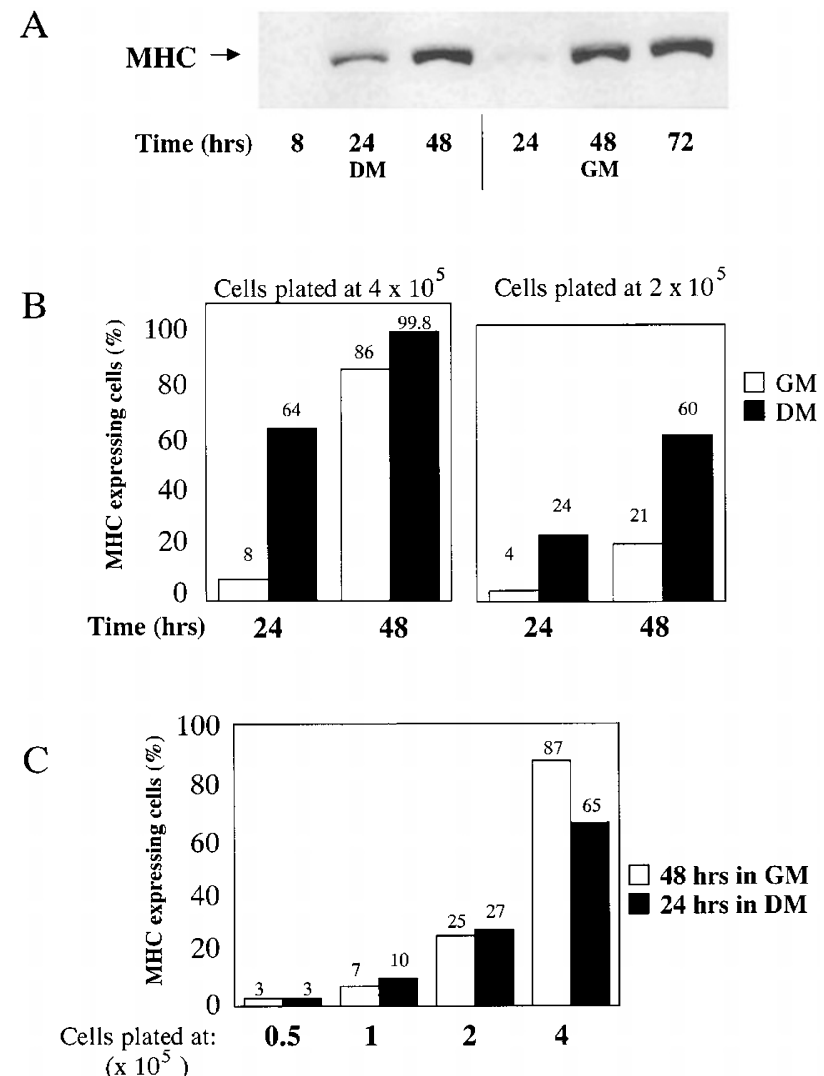

Figure 1 Differentiation is a density- and time-dependent process. (A) 23A2 myoblasts were plated at $4 \times 10^{5}$ and the next day switched to either fresh GM or DM for the indicated times prior to lysis. Whole cell extracts were prepared and $100 \mu \mathrm{g}$ of protein from each extract was separated by SDS-PAGE (8\%). Following electrophoretic transfer, a Western analysis was performed for MHC as described in Materials and Methods. Shown are results from one experiment which are representative of three independent experiments. (BC) $23 \mathrm{~A} 2$ myoblasts were plated as indicated and the next day switched to either fresh GM or DM for the indicated times. Cells were immunochemically stained for MHC expression and counted as described in Materials and Methods. Values represent the per cent of attached cells that express MHC and do not reflect the cells that have fallen off the plate after completing the process of apoptosis. Shown in each are the average of duplicates (variation $<5 \%)$ for one experiment that are representative of two independent experiments 
A

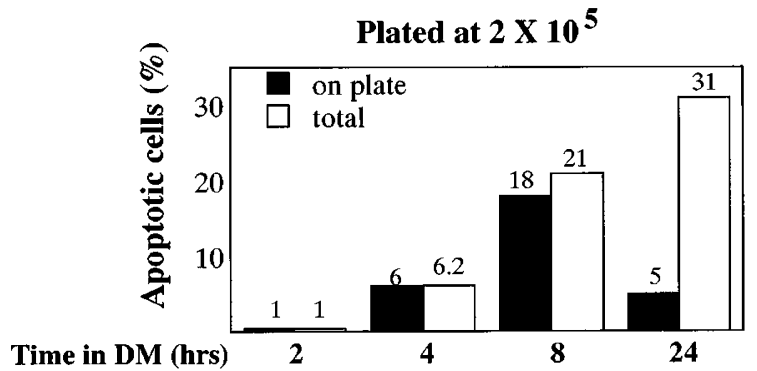

B

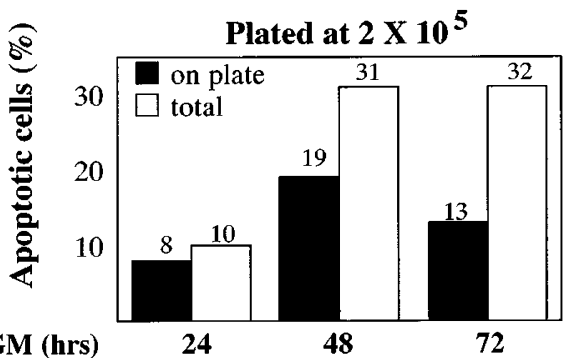

$\mathrm{C}$

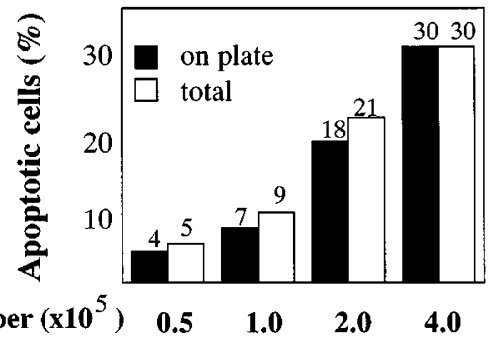

$\mathrm{D}$

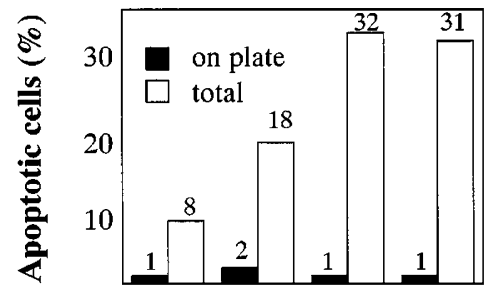

24 hrs in DM

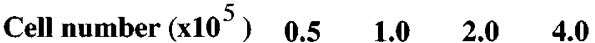

$\mathrm{E}$

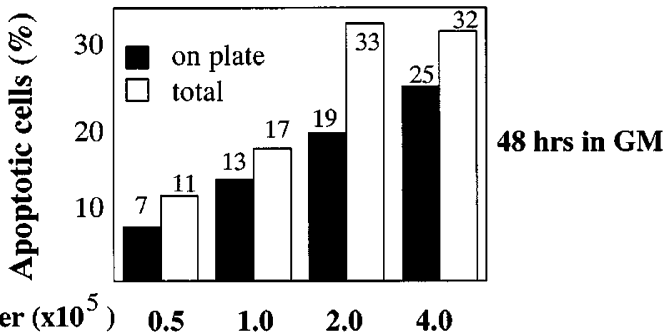

Cell number $\left(\mathrm{x} 10^{5}\right) \quad 0.5 \quad 1.0 \quad 2.0 \quad 4.0$

Figure 2 In situ DNA fragmentation is a density- and time-dependent process. (A-E) $23 \mathrm{~A} 2$ myoblasts were plated as indicated and the next day switched to either fresh GM or DM for the indicated times. In situ DNA fragmentation was visualized using apoTACS ${ }^{T M}$ from R\&D Systems per manufacturer's instructions and counted as described in Materials and Methods. Values represent the percentage of cells both attached and floating that display in situ DNA fragmentation. Shown in each are the results from one experiment that are representative of two independent experiments

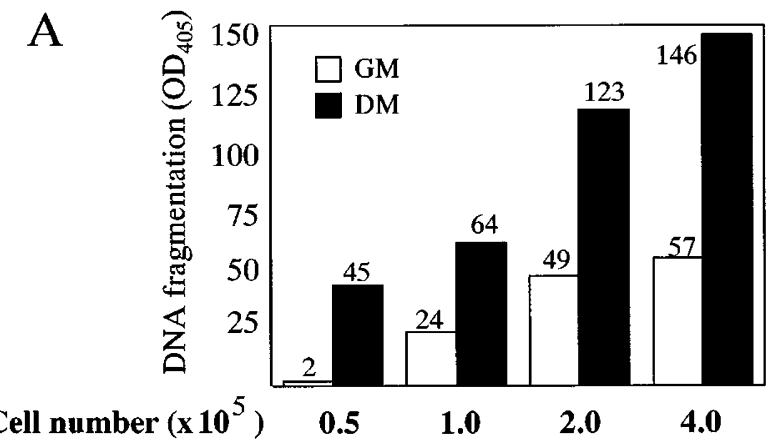

B

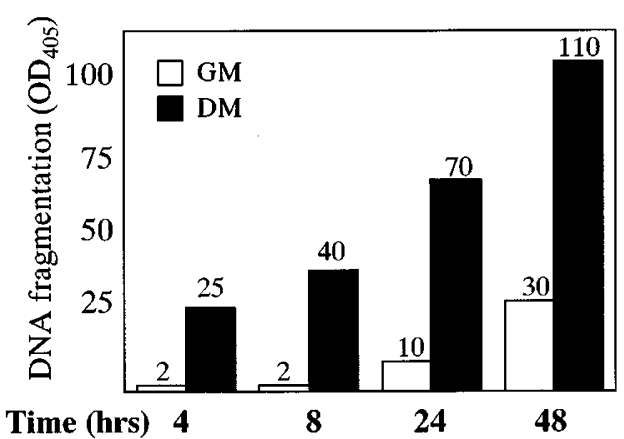

Figure 3 The appearance of cytosolic nucleosomes is a density- and timedependent process. In (A) 23A2 myoblasts were plated as indicated and the next day switched to either fresh GM or DM for $8 \mathrm{~h}$. In (B) 23A2 myoblasts were plated at $5 \times 10^{4}$ and the next day switched to either fresh GM or DM for the indicated times. Cytosolic nucleosomes were measured using the Cell Death Detection ELISA Plus Kit (Roche Diagnostics) per manufacturer's instructions within the linear range of the assay. Absorbance was normalized by cell number as described in Materials and Methods

takes into account both cells that remain on the plate and those that have fallen off of the plate after completing the process of apoptosis. By plating cells at the same density and monitoring DNA fragmentation over time in either DM (Figure 2A) or GM (Figure 2B), we can document that apoptosis is time dependent. By plating cells at different densities and monitoring DNA fragmentation after the same time in either DM (Figure 2C, D) or GM (Figure 2E), we can also show that apoptosis is density dependent. It is clear from both analyses that these variables of time and density are inversely related and that maximally $30-33 \%$ of the culture apoptoses. Once again, it is also clear that apoptosis occurs faster when cells are switched from GM to DM than under extended culture in GM, for example, compare $24 \mathrm{~h}$ in DM to $24 \mathrm{~h}$ in GM (Figure 2A, B). The lag time between the apoptosis that occurs under extended culture in GM and the apoptosis that occurs when cells are switched from GM to DM is roughly $20 \mathrm{~h}$ (compare $4 \mathrm{~h}$ Figure $2 \mathrm{~A}$ to $24 \mathrm{~h}$ Figure $2 \mathrm{~B}$ ). The lag time between the appearance of DNA fragmentation and detachment from the plate is less than $16 \mathrm{~h}$ in DM (Figure $2 \mathrm{C}, \mathrm{D}$ ) and greater than $24 \mathrm{~h}$ in GM (Figure 2B, compare $48 \mathrm{~h}$ to $72 \mathrm{~h}$ ). We have also shown that the process of apoptosis, as monitored by DNA fragmentation and including detachment from the plate, can be completed much faster than the process of differentiation. This is most clearly observed by 
plating cells at $4 \times 10^{5}$ followed by culture for $24 \mathrm{~h}$ in DM prior to the detection of either differentiation (Figure $1 \mathrm{~A}$ ) or apoptosis (Figure 2D). Although the percentage of cells destined to apoptose is decided after $8 \mathrm{~h}$ in DM or $48 \mathrm{~h}$ in $\mathrm{GM}$, these apoptotic cells have not yet detached from the plate under these conditions. This raises the possibility that the amount of MHC (since lysates are equalized for total cell protein) and the percentage of cells expressing $\mathrm{MHC}$ are slightly underestimated under conditions where apoptosis is not complete or is complete but the cells have yet to detach from the plate.

The ELISA analysis is quantitative with respect to the amount of cytosolic nucleosomes in cells remaining on the plate. Since after $8 \mathrm{~h}$ in DM most apoptotic cells are still attached to the plate (Figure $2 \mathrm{C}$ ), this time point was chosen to demonstrate the density dependence of DNA fragmentation in either GM or DM (Figure 3A). To demonstrate that DNA fragmentation is also time dependent, myoblasts were plated at $5 \times 10^{4}$ and then cultured in either GM or DM for various times (Figure 3B). After $8 \mathrm{~h}$ in $\mathrm{DM}$, the percentage of apoptotic cells correlates roughly with the amount of cytosolic nucleosomes (compare Figure $2 \mathrm{C}$ with Figure $3 \mathrm{~A}$ ). This is not true, however, as the process of apoptosis progresses over time. After $8 \mathrm{~h}$, apoptotic cells equaling $4 \%$ (Figure $2 \mathrm{C}$ ) of the population contain 40-45 absorbance units of cytosolic nucleosomes (Figure 3A, B) while after $24 \mathrm{~h}$ in DM apoptotic cells equaling $1 \%$ (Figure 2D) of the population contain 70 absorbance units of cytosolic nucleosomes (Figure 3B). Since the total cell number does not change between 8 and $24 \mathrm{~h}$ in DM, we can calculate that myoblasts after $24 \mathrm{~h}$ in DM contain almost ninefold more cytosolic nucleosomes than myoblasts after $8 \mathrm{~h}$ in DM. Nonetheless, it is again clear from both analyses that apoptosis occurs faster when cells are switched from GM to DM than under extended culture in GM and that apoptosis depends on the variables of cell density and time and that these variables are inversely related.

\section{Abrogation of differentiation can be separated from abrogation of apoptosis}

We have rigorously demonstrated that under various conditions of depleted growth factors some skeletal myoblasts differentiate while others apoptose. Even though initiated by the same stimulus of growth factor depletion, it is still possible that these processes can be separated. To examine this possibility, we measured apoptosis in two differentiation-defective myoblast cell lines. Myoblasts expressing constitutively active Ras (A2:H-Ras myoblasts) are differentiation-defective ${ }^{29,30}$ (Figure 4A) and apoptose at a level less than $1 / 10$ that of the parental $23 A 2$ myoblasts in either GM (Figure 4B) or DM (Figure 4B-D). In contrast, the apoptosis of myoblasts $(A 2: E 1 A)$ rendered differentiation-defective (Figure 4A) by expression of $E 1 A^{31}$ is slightly elevated when compared to parental 23A2 myoblasts in either GM (Figure 4B) or DM (Figure 4B-D). The fact that $A 2: E 1 A$ myoblasts are differentiation-defective yet are not impaired in their ability to apoptose indicates that the positive effect of constitutively active Ras on myoblast
A

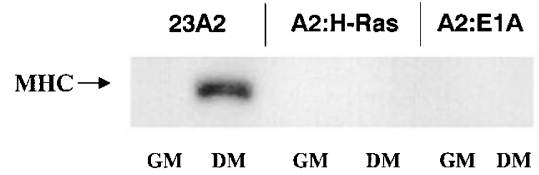

B

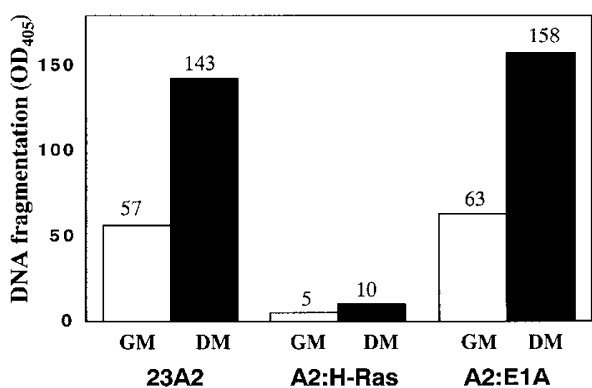

$\mathrm{C}$

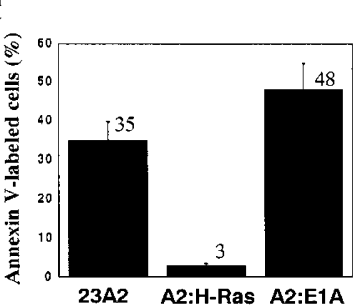

$\mathrm{D}$

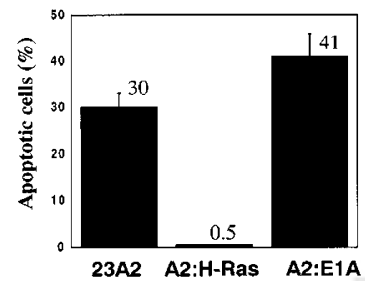

Figure 4 Abrogated apoptosis in $\mathrm{A} 2 \mathrm{H}$-Ras myoblasts but not in $\mathrm{A} 2 \mathrm{E}$ 1A myoblasts. (A) 23A2, A2:H-Ras, and A2:E1A myoblasts were plated at $4 \times 10^{5}$ and the next day switched to either fresh GM or DM for $24 \mathrm{~h}$ prior to the determination of MHC expression as described for Figure 1. (B) 23A2, A2: HRas, and A2:E1A myoblasts $\left(2 \times 10^{5}\right)$ were plated and the next day switched to either fresh GM or DM for $8 \mathrm{~h}$. Cytosolic nucleosomes were measured as described for Figure 3. Shown are the average of duplicates for one experiment that are representative of two independent experiments. (C, D) 23A2, A2:H-Ras, and A2:E1A myoblasts were plated at $4 \times 10^{5}$ and the next day switched to DM for $12 \mathrm{~h}$ (C) and $24 \mathrm{~h}$ (D) prior to the determination of (C) PS asymmetry via annexin V labeling as described in Materials and Methods or (D) in situ DNA fragmentation as described for Figure 2. In (C) values represent those cells that labeled with annexin $\mathrm{V}$ but not with propidium iodide and are the average of duplicate experiments

survival is not merely a consequence of its negative effect on their differentiation.

Signaling by Ras through either the PI3-kinase/Akt pathway $^{32}$ or the Raf/MEK/MAPK pathway has been implicated in cell survival. ${ }^{33}$ Since PI3 kinase/Akt signaling has been implicated in the normal survival/differentiation of skeletal myoblasts, ${ }^{20,21,23,24}$ we focused on the role of Raf/ MEK signaling in the increased survival of $\mathrm{A} 2 \mathrm{H}$-Ras myoblasts. We have previously reported that PD 098059, a selective MEK inhibitor, does not affect the differentiation of skeletal myoblasts. ${ }^{29}$ Furthermore, while PD 098059induced abrogation of constitutive MEK signaling in $\mathrm{A} 2: \mathrm{H}$ Ras myoblasts reverts their transformed phenotype, this does not revert their differentiation-defective phenotype. ${ }^{29}$ We have now determined that PD 098059-induced abrogation of constitutive MEK signaling (Figure 5A) does appear to increase the amount of DNA fragmentation in apoptotic A2:H-Ras myoblasts roughly 4-6-fold (Figure 5B). PD 098059 treatment, however, induces only a modest increase in the per cent of apoptotic A2:H-Ras myoblasts 
A

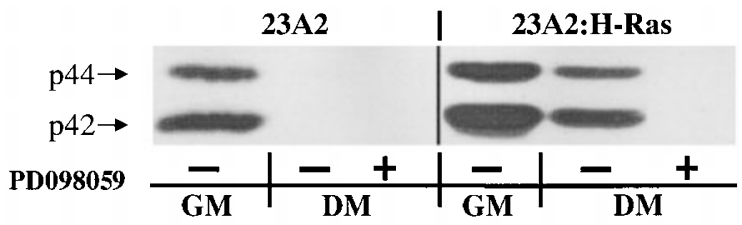

B

$\mathrm{C}$
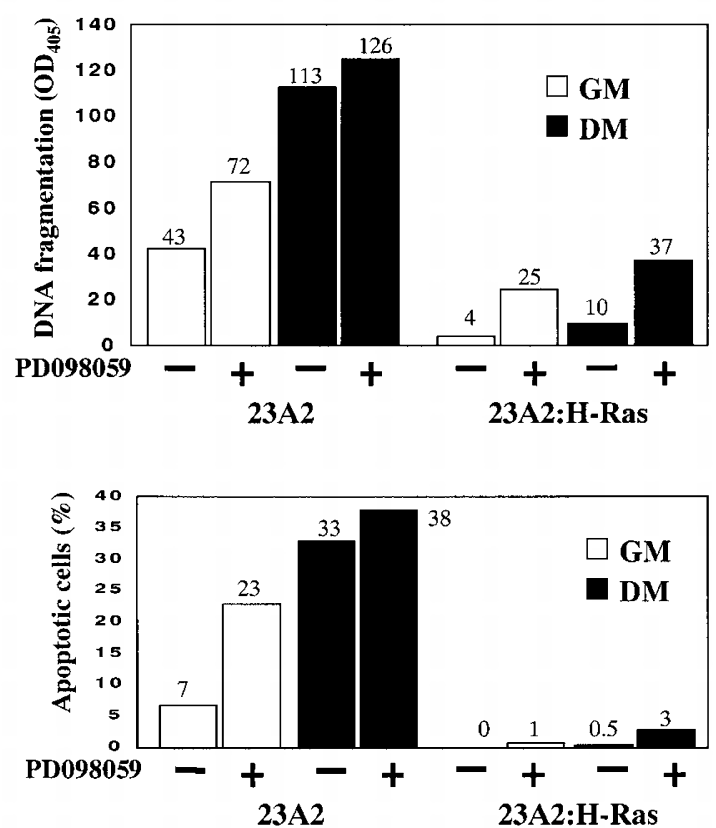

Figure 5 Abrogated apoptosis in A2:H-Ras myoblasts is independent of constitutive MEK signaling to MAPK. Equal numbers $\left(2 \times 10^{5}\right)$ of $23 \mathrm{~A} 2$ and A2: $\mathrm{H}$-Ras myoblasts were plated and the next day switched to either fresh GM or DM with or without $50 \mu \mathrm{M}$ PD098059 for $8 \mathrm{~h}$. In (A), whole cell extracts were prepared and $100 \mu \mathrm{g}$ of protein from each extract was separated by SDSPAGE $(10 \%)$. Following electrophoretic transfer, a Western analysis was performed for phospho-MAPK as described in Materials and Methods. Shown are results from one experiment which are representative of three independent experiments. In (B), cytosolic nucleosomes were measured as described for Figure 3. Shown are the average of duplicates (variation $<2 \%$ ) for one experiment that are representative of three independent experiments. In (C) apoptotic cells were assessed as described in Figure 2

(Figure 5C). While constitutive MEK signaling may play a role in DNA fragmentation in apoptotic A2:H-Ras myoblasts, the role of constitutive MEK signaling in the overall survival of A2:H-Ras myoblasts is minimal. Even when cultured in DM plus PD 098059 where the greatest apoptosis of $\mathrm{A} 2 \mathrm{H}$-Ras myoblasts is observed, the per cent apoptotic A2:H-Ras myoblasts is less than $1 / 10$ that of apoptotic 23A2 myoblasts cultured in DM without PD 098059 (Figure 5C).

The concentration of PD 098059 used to completely abrogate the constitutive MEK signal in A2:H-Ras myoblasts reduced the serum stimulated MEK signal by roughly $80 \%$ (data not shown). Interestingly, this reduction of the serum-stimulated MEK signal in 23A2 myoblasts caused the level of DNA fragmentation (Figure $5 \mathrm{~B}$ ) and the percentage of apoptotic cells (Figure $5 \mathrm{C}$ ) to approach that observed when $23 \mathrm{~A} 2$ myoblasts are cultured in DM. This suggests that serum-stimulated

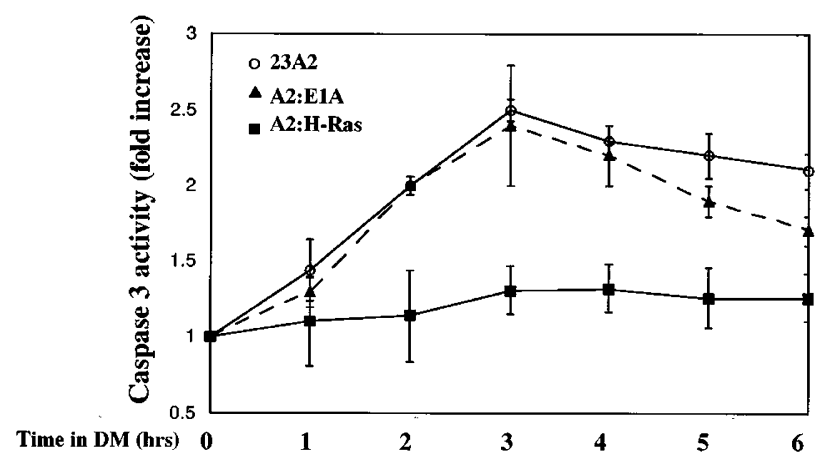

Figure 6 Activation of caspase 3 in $23 A 2$ and $A 2: E 1 A$, but not in $A 2: H-R a s$ myoblasts. Equal numbers of 23A2, $\mathrm{A} 2: \mathrm{H}-\mathrm{Ras}$, and $\mathrm{A} 2: \mathrm{E} 1 \mathrm{~A}$ myoblasts were plated and the next day switched to either fresh GM or DM for the indicated times. Caspase 3 colorimetric assays were performed using the Caspase 3 Colorimetric Assay Kit from R\&D Systems per manufacturer's instructions. Absorbance was normalized by the protein concentration of each lysate. Fold increase in caspase activity in DM was calculated relative to the absorbance value obtained from the lysate of cells incubated in fresh GM for $1 \mathrm{~h}$. Shown are the average of duplicates for one experiment that are representative of two independent experiments

MEK signaling may play a critical role in myoblast survival in GM.

\section{Abrogation of caspase 3 signaling delays apoptosis without affecting differentiation}

Caspase 3 , along with caspases 6 and 7 , are considered to be the primary downstream caspases ultimately responsible for carrying out the process of apoptosis. Caspase 3-independent apoptosis, however, has been reported in several systems. ${ }^{34,35}$ Since no reports have yet examined the activation of caspases when skeletal myoblasts are induced to differentiate, we measured the activation of caspase 3 when 23A2 myoblasts are cultured in DM. We found that caspase 3 is activated with maximal activation of roughly $240 \%$ occurring after $3 \mathrm{~h}$ in DM (Figure 6). Similar results were obtained when $\mathrm{A} 2 \mathrm{E} 1 \mathrm{~A}$ myoblasts were cultured in DM. A minimal $(30 \%)$ activation of caspase 3 is observed when A2: $\mathrm{H}$-Ras myoblasts are cultured in DM for $3 \mathrm{~h}$ (Figure 6). To determine the importance of caspase 3 activation to the apoptosis of 23A2 myoblasts cultured in DM, we used the selective pharmacological inhibitor DEVD-fmk. Since caspase 3 is maximally activated in 23A2 myoblasts cultured in $\mathrm{DM}$ for $3 \mathrm{~h}$ (Figure 6), we initially chose this condition to examine the effect of DEVD-fmk on caspase 3 activation. We determined that DEVD-fmk blocked caspase 3 activation in a dose-dependent manner (Figure 7A). We then determined the effect of DEVD-fmk on the DNA fragmentation of $23 \mathrm{~A} 2$ myoblasts after $8 \mathrm{~h}$ in DM, a time point at which we had previously determined that most apoptotic myoblasts are still attached to the plate (Figure $2 \mathrm{C}$ ). We found that DNA fragmentation can be completely blocked with only a $64 \%$ reduction in caspase 3 activity (Figure 7A). Furthermore, no apoptotic myoblasts were detected at this time point in the presence of DEVD-fmk (data not shown).

We have used the A2:E1A myoblasts to determine that the inhibition of differentiation does not always result in the 
A

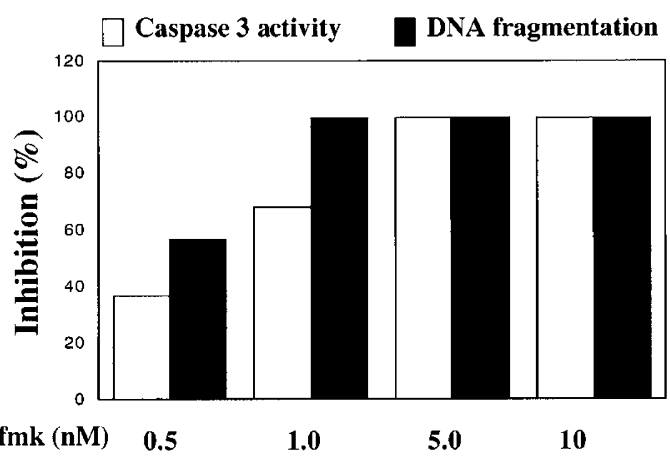

B

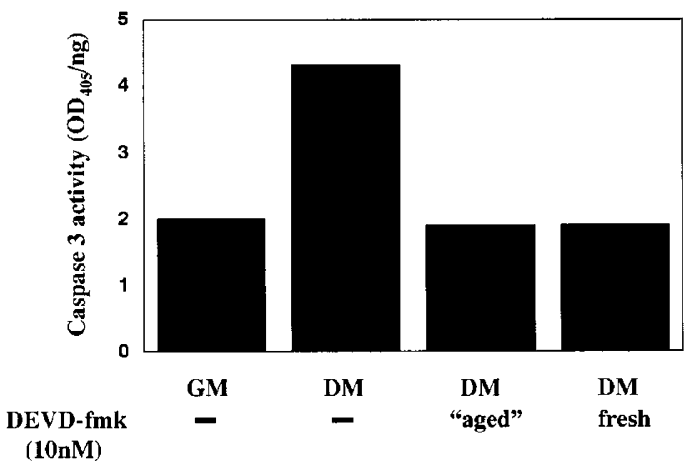

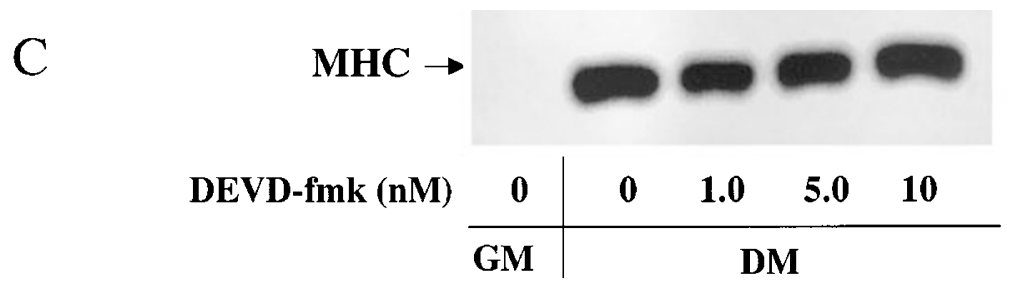
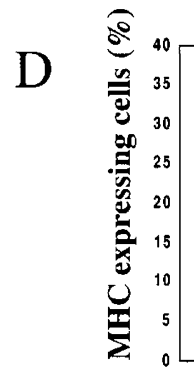

DEVD-fmk (10nM)

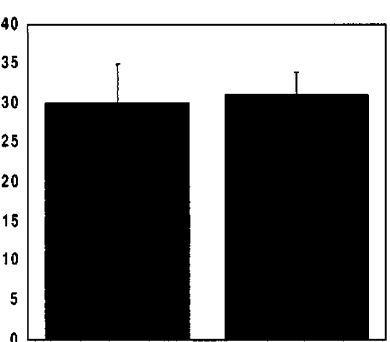

$+$

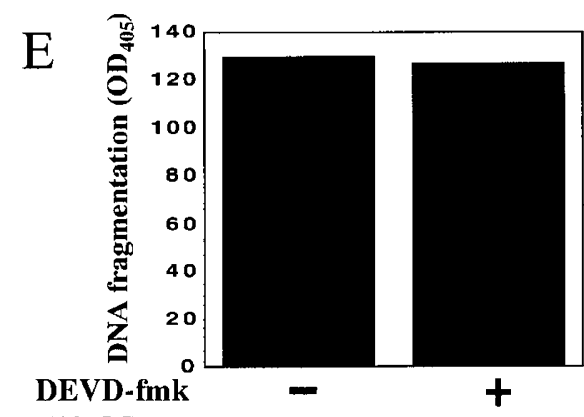

(10nM)

$\mathrm{F}$

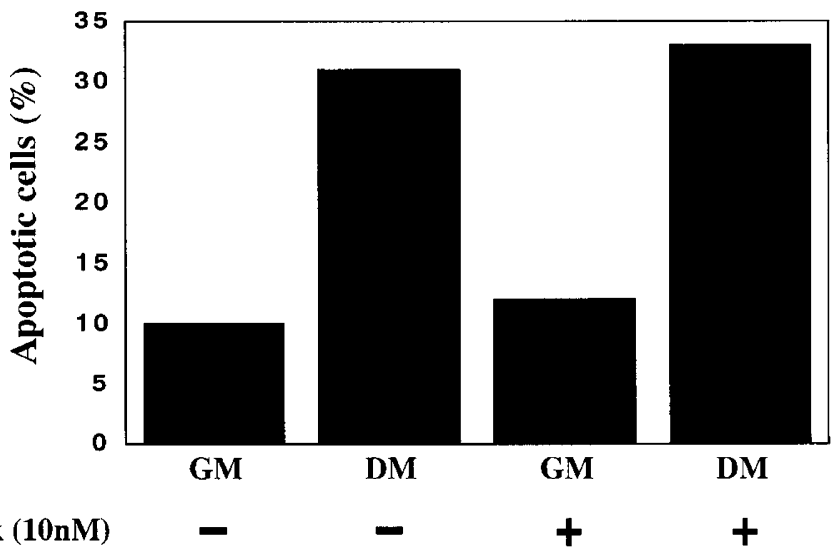

Figure 7 Inhibition of caspase 3 activity abrogates apoptosis but not differentiation. (A) Equal numbers of 23A2 myoblasts were plated and the next day switched to DM with or without the indicated concentration of DEVD-fmk for $3 \mathrm{~h}$ prior to the determination of caspase 3 activity or $8 \mathrm{~h}$ prior to the determination of cytosolic nucleosomes. Absorbance values for each were normalized as in Figures 3 and 6 , respectively. Per cent inhibition with DEVD-fmk was calculated relative to values obtained without DEVD-fmk. (B) Equal numbers of $23 \mathrm{~A} 2$ myoblasts were plated and the next day switched to either fresh GM, fresh DM with or without $10 \mathrm{nM}$ DEVD-fmk, or DM with $10 \mathrm{nM}$ DEVD-fmk that had been 'aged' for $24 \mathrm{~h}$ (see Results). Cells were lysed after $3 \mathrm{~h}$ and caspase 3 activity was determined as described in Figure 6. In (C-F), equal numbers of $23 \mathrm{~A} 2$ myoblasts were plated and the next day switched to fresh GM or DM with or without the indicated concentration of DEVD-fmk for $24 \mathrm{~h}$ prior to the determination of (C) MHC expression as described in Figure 1A, (D) the percentage of myoblasts expressing MHC as described in Figure 1B, (E) DNA fragmentation as described in Figure 3, and (F) the percentage of apoptotic cells as described in Figure 2 
inhibition of apoptosis suggesting that the signaling pathways responsible for differentiation can be separated from those responsible for apoptosis. To further establish that point, we chose to monitor the effect of DEVD-fmk on $\mathrm{MHC}$ expression in $23 \mathrm{~A} 2$ myoblasts. MHC, however, can only be readily detected after $24 \mathrm{~h}$ in DM, a time point at which all myoblasts that have apoptosed have detached from the plate. Consequently, caspase 3 activity is not detected after $24 \mathrm{~h}$ in DM (data not shown). To assess the stability of DEVD-fmk, we cultured myoblasts in DM with DEVD-fmk for $24 \mathrm{~h}$ to create 'aged' media. We then switched naïve 23A2 myoblasts from GM to this 'aged' media and measured the activation of caspase 3 after $3 \mathrm{~h}$, since this is the time point at which maximal caspase 3 activity is observed. We found that the caspase 3 inhibitory activity of 'aged' DEVD-fmk was comparable to that of 'fresh' DEVD-fmk (Figure 7B). Having assured ourselves of the stability of DEVD-fmk, we next assessed the effect of DEVD-fmk on the differentiation of $23 \mathrm{~A} 2$ myoblasts and found no effect on the expression of $\mathrm{MHC}$ (Figure 7C) or the percentage of myoblasts expressing MHC (Figure 7D). Finally, we assessed the effect of DEVD-fmk on the apoptosis of $23 A 2$ myoblasts cultured in DM for $24 \mathrm{~h}$. After $24 \mathrm{~h}$, DEVD-fmk, although still able to abrogate caspase 3 activity, no longer inhibited DNA fragmentation (Figure 7E) and did not reduce the percentage of apoptotic myoblasts (Figure 7F). This result indicates that abrogating caspase 3 activity only serves to delay the apoptosis of $23 \mathrm{~A} 2$ myoblasts and eliminates caspase 3 as a target that can be manipulated to block apoptosis without affecting differentiation.

\section{Discussion}

In several cell types, differentiation and apoptosis are coordinately regulated. ${ }^{1,2,4,5}$ While undoubtedly a vital component of development, the linkage of these two processes is obviously detrimental ${ }^{12,13}$ to the use of myoblast transfer as a gene complementation approach for the treatment of muscular dystrophies ${ }^{8-11}$ and certain metabolic deficiencies. ${ }^{36}$ Finding targets to manipulate that would allow abrogation of apoptosis without affecting differentiation could improve the efficacy of these approaches. We have shown that in skeletal myoblasts both differentiation and the accompanying apoptosis depend on the variables of cell density and time and that these variables are inversely related. For many years it has been accepted that skeletal myoblast differentiation depends on both growth factor depletion and the autocrine secretion of IGFs. ${ }^{18,19}$ This would explain the dependence of differentiation on the variables of cell density and time and the inverse relationship of these variables. The greater the cell density the faster the growth factor depletion in GM. Likewise, the greater the cell density the less time needed to secrete a sufficient concentration of IGFs in either GM or DM. Obviously, the signaling pathways regulating survival and differentiation are necessarily linked. In fact, survival may be functionally synonymous with differentiation. Whether survival permits differentiation or differentiation permits survival is not clear and in fact, either may be true.
There are at least two scenarios to explain the fact that apoptosis, like differentiation, is also dependent on the variables of cell density and time. The first is simply that, while differentiation is initiated by growth factor depletion, apoptosis may be initiated by trophic factor depletion. Whether or not these factors are the same or distinct remains to be determined. The second is that apoptosis may depend on the secretion of an autocrine factor, which by definition would be a death ligand. It is even possible that both scenarios are responsible for the apoptosis that accompanies skeletal myoblast differentiation.

Constitutive signaling by Ras abrogates the differentiation of skeletal myoblasts. ${ }^{29,30,37,38}$ We demonstrate in this study that the apoptosis of differentiation-defective $\mathrm{A} 2: \mathrm{H}-$ Ras myoblasts is greatly impaired when compared to parental 23A2 myoblasts cultured under similar conditions. The apoptosis of myoblasts rendered differentiation-defective by expression of E1A (A2:E1A myoblasts), however, is not impaired under these same conditions and indicates that the diminished apoptosis of $\mathrm{A} 2 \mathrm{H}$-Ras myoblasts is not merely a consequence of their inability to differentiate. If Ras is signaling through only one pathway to inhibit both differentiation and apoptosis, then the signaling pathway(s) leading to differentiation or apoptosis might initially be the same pathway. The fact that the apoptosis of differentiation-defective E1A myoblasts is not diminished suggests that at some point this pathway bifurcates and that $E 1 A$ is inhibiting only the pathway that leads to differentiation. Alternatively, Ras may be signaling through one pathway to inhibit differentiation and another pathway to inhibit apoptosis. This possibility is currently being investigated with the hope of identifying a pathway downstream from Ras that can be manipulated to inhibit the apoptosis of skeletal myoblasts without affecting their differentiation.

The best characterized signaling pathways downstream from Ras are PI3-kinase/Akt and the Raf/MEK. ${ }^{39}$ Since signaling through the PI3-kinase pathway has been shown to be critical for normal skeletal myoblast survival/differentiation, ${ }^{20,21,23,24}$ and Ras signaling abrogates differentiation, ${ }^{29,30,37,38}$ we focused our attention on the Raf/MEK pathway which has been shown to be anti-apoptotic. ${ }^{33} \mathrm{We}$ have shown that signaling through MEK may play a role in the survival of myoblasts in GM since the abrogation of MEK signaling increases apoptosis in GM to almost the level observed when myoblasts are cultured in DM. The cells that die as a consequence of inhibiting MEK may be the same cells destined to die during the process of differentiation. If this is true, since constitutive MEK signaling does not abrogate differentiation, ${ }^{40}$ then MEK may be a target which can be manipulated to abrogate apoptosis without affecting differentiation. Experiments to determine this are underway. We have also found that inhibiting constitutive MEK signaling is not sufficient to permit an apoptotic response in A2:H-Ras myoblasts comparable to that of parental 23A2 myoblasts.

With the hope of finding targets that can be manipulated to abrogate apoptosis without affecting the differentiation of skeletal myoblasts, we next examined the activation of caspase 3 . We have determined that caspase 3 is activated 2.4-fold when 23A2 myoblasts are cultured in DM. 
Interestingly, caspase 3 is modestly (30\%) activated in parallel cultures of $\mathrm{A} 2: \mathrm{H}-$ Ras myoblasts suggesting that a small amount of caspase 3 activation is tolerated. Selective pharmacological inhibitors of caspase signaling are available and have been used not only to assess the significance of caspase activation in tissue culture but also in animal models of stroke, myocardial ischemia-reperfusion injury, liver disease and traumatic brain injury. ${ }^{26}$ We have used the selective caspase 3 inhibitor, DEVD-fmk, to determine that activation of caspase 3 plays a role in $23 \mathrm{~A} 2$ myoblast apoptosis since pharmacological abrogation of caspase 3 activity inhibits apoptosis after $8 \mathrm{~h}$ in DM. The fact that total inhibition of DNA fragmentation can be achieved with only a $64 \%$ reduction in caspase 3 activity further suggests that a small amount of caspase activity can be tolerated. The inhibition achieved through treatment with DEVD-fmk, however, is not sustainable. Although treatment with DEVD-fmk does not affect differentiation, the fact that it only serves to delay skeletal myoblast apoptosis for a short time eliminates caspase 3 as a target for therapeutic manipulation to improve the efficacy of myoblast transfer. Experiments are underway to further characterize the apoptotic pathway in 23A2 myoblasts and to determine at what point in that pathway Ras is exerting its anti-apoptotic effect.

\section{Materials and Methods}

\section{Cells and cell culture}

The growth and differentiation properties of 23A2 myoblasts and the 23A2 derivatives expressing $\mathrm{G} 12 \mathrm{~V}: \mathrm{H}-\mathrm{Ras}$ (A2:H-Ras myoblasts) or E1A (A2:E1A) have been reported previously. ${ }^{24-26}$ All cells were cultured at low density on gelatin-coated plates and maintained in growth medium (GM), which consists of basal modified Eagle's medium (BME), $10 \%$ fetal bovine serum (FBS), and a $1 \%$ combination of 10000 I.U./ml penicillin and $10000 \mu \mathrm{g} / \mathrm{ml}$ streptomycin (1\% P/S). Differentiation was induced by switching cells from GM to differentiation medium (DM), which consists of BME, $1 \% \mathrm{P} / \mathrm{S}$ and $0 \% \mathrm{FBS}$. Cells were incubated at $37^{\circ} \mathrm{C}$ in $5 \% \mathrm{CO}_{2}$. PD098059 and DEVD-fmk were each dissolved in DMSO to give a final concentration of $37 \mathrm{mM}$ and $10 \mu \mathrm{M}$ respectively prior to storage at $-20^{\circ} \mathrm{C}$. Appropriate volumes of DMSO alone $(0.15 \%$ $\mathrm{v} / \mathrm{v}$ ) were added to control cultures and never exceeded $0.14 \% \mathrm{v} / \mathrm{v}$.

\section{Immunoblot analysis}

Cells were plated on $35 \mathrm{~mm}$ dishes and the next day switched to fresh GM or DM for various times prior to being rinsed in cold $1 \times$ phosphate buffered saline (PBS) and then lysed in p21 lysis buffer (20 mM MOPS $\mathrm{pH}$ 7.4, $4 \mathrm{mM}$ magnesium chloride, $200 \mathrm{mM}$ sucrose, $0.1 \mathrm{mM}$ EDTA, $0.001 \%$ DNAse, $1 \mathrm{M}$ phenyl methyl sulfonyl fluoride, and $50 \mu \mathrm{g} / \mathrm{ml}$ each of aprotinin, pepstatin, and leupeptin) with $1 \%$ CHAPS. The protein concentrations of all lysates were determined using Coomassie Protein Assay Reagent from Pierce as per manufacturer's instructions. Following protein determination, lysates $(50 \mu \mathrm{g}$ for $\mathrm{MHC}$ detection and $100 \mu \mathrm{g}$ for phospho-MAPK) were denatured in $5 \times$ sample buffer (10\% SDS, $50 \%$ glycerol, $10 \%$ 2-mercaptoethanol, $\mathrm{pH} 6.8)$ and electrophoresed through denaturing polyacrylamide gels ( $8 \%$ for MHC detection and 10\% for phospho-MAPK). Following SDS polyacrylamide gel electrophoresis (SDS-PAGE), samples were transferred electrophoretically for four amp hours to Hybond-P polyvinylidene difluoride membranes in transfer buffer containing $80 \%$ methanol and $1 \mathrm{~g} / \mathrm{l} \mathrm{SDS}$. Membranes were dried at $37^{\circ} \mathrm{C}$ for $30 \mathrm{~min}$. Western analysis was performed to detect MHC by incubating the membranes with a mouse monoclonal antibody MF20 that is specific for skeletal myosin heavy chain protein for $1 \mathrm{~h}$ followed by an HRP-conjugated mouse polyclonal antibody diluted $1: 1000$ for $1 \mathrm{~h} .{ }^{29}$ Western analysis to detect phospho-MAPK was performed with a polyclonal antibody to the phosphorylated form of MAPK (Santa Cruz) diluted 1:500 and incubated with the membrane overnight followed by an HRP-conjugated mouse polyclonal antibody diluted 1:1000 and incubated with the membrane for $1 \mathrm{~h}$. To ensure equal protein loading, Western analysis was performed on parallel membranes using an antiMAPK mouse polyclonal antibody (Upstate Biotechnologies) and an HRP-conjugated anti-mouse polyclonal antibody, each diluted 1:1000 and incubated with the membranes for $1 \mathrm{~h}$. After each incubation with antibody and prior to the addition of chemiluminescent substrate, membranes were washed five times in $1 \times$ TBS (tris-buffered saline $\mathrm{pH} 7.4$ ) with $1 \%$ NP-40. Membranes were then incubated with SuperSignal West Pico Chemiluminescent Substrate (Pierce) for $60 \mathrm{~s}$ and bands were visualized using either Kodak Scientific Imaging Film or a Bio-Rad Fluor-S Multi-imager.

\section{Immunohistochemistry}

Cells were plated on gridded $35 \mathrm{~mm}$ culture dishes and the next day switched to fresh GM or DM for various times. Attached cells were rinsed in PBS and fixed. Fixed cells were permeabilized with trisbuffered saline/0.1\% NP40 and blocked with $2 \%$ horse serum in PBS. This was followed by incubation with the MF20 antibody for $60 \mathrm{~min}$, washing with PBS and incubation with a biotinylated goat anti-mouse antibody per manufacturer's instructions (Santa Cruz: ABC staining system). After washing, cells were incubated with HRP-streptavidin and immunoreactivity was visualized using 3,3'-diaminobenzidine (DAB), $\mathrm{H}_{2} \mathrm{O}_{2}$ and $\mathrm{NiCl}_{2}$ per manufacturer's instructions. For each condition, at least two fields of not less than 100 cells each were counted independently by two investigators without prior knowledge of the sample identity.

\section{Apoptosis assays}

TUNEL assay: DNA fragmentation is a commonly used marker for apoptotis. Cells were plated on gridded $35 \mathrm{~mm}$ culture dishes and the next day switched to fresh GM or DM for various times prior to being rinsed in PBS. In situ DNA fragmentation was visualized using apoTACS $^{T M}$ from R\&D Systems per manufacturer's instructions. Floating cells were treated as suspension cells per manufacturer's instructions. Briefly, floating cells were collected by centrifugation at $500 \times g$ for $5 \mathrm{~min}$, washed and resuspended in $3.7 \%$ buffered formaldehyde for $10 \mathrm{~min}$. After centrifugation, floating cells were resuspended in $80 \%$ ethanol and spotted onto new gridded $35 \mathrm{~mm}$ culture dishes. After drying for $2 \mathrm{~h}$, cells were immersed in $70 \%$ ethanol for $10 \mathrm{~min}$ and then allowed to dry. After washing, attached cells were fixed in $3.7 \%$ buffered formaldehyde for $10 \mathrm{~min}$, washed, and dehydrated through incubation in increasing concentration of ethanol $(70 \%, 80 \%, 95 \%$ and $100 \%)$ for 5 min intervals separated by 10 min intervals of drying. Both dehydrated floating cells and attached cells were rehydrated by immersing for 5 min each in 100\%, 95\% then $70 \%$ ethanol. Cells were then incubated in $1 \times$ PBS for $5 \mathrm{~min}$ and permeabilized with proteinase $\mathrm{K}$. After washing, cells were incubated for $5 \mathrm{~min}$ in terminal deoxynucleotidyl transferase enzyme (TdT) labeling buffer followed by incubation with labeling reaction mix (TDT 
plus B-dNTPs in labeling buffer) for $1 \mathrm{~h}$ in a humidity chamber. Cells were then incubated with TdT stop buffer and washed prior to incubation in detection mix (anti-BrdU antibody and streptavidin-HRP) for $1 \mathrm{~h}$. After washing, cells were incubated with TACS blue label and washed prior to final viewing. For each condition, at least two fields of not less than 100 cells each were counted independently by two investigators without prior knowledge of the sample identity. Total cell numbers were also determined for both attached and corresponding floating cells from parallel plates. The total per cent of apoptotic cells for each plate was calculated as follows:

$\{[($ total floating cells $\times$ per cent stained $)+$ (total attached cells $\times$ per cent stained)] divided by (total floating cells + total attached cells) $\} \times 100$

Cytosolic nucleosome ELISAs: DNA fragmentation and nuclear membrane disruption, as monitored by the presence of cytosolic nucleosomes, was also used as a marker for apoptosis. Cytosolic nucleosomes were measured using the Cell Death Detection ELISA Plus Kit (Roche Diagnostics) per manufacturer's instructions. Cells were plated on $35 \mathrm{~mm}$ dishes and the next day switched to fresh GM or DM for various times. Attached cells were rinsed in PBS and then lysed by incubation in $300 \mu \mathrm{l}$ cell lysis buffer from the kit on a rocker for $30 \mathrm{~min}$ at room temperature. Samples were diluted fourfold and $20 \mu \mathrm{l}$ of each sample was transferred to a 96-well, flatbottomed, streptavidin-coated microtiter plate. Seventy-two microliters of $1 \times$ incubation buffer from the kit, and $4 \mu \mathrm{l}$ each of biotin conjugated anti-histone antibody and peroxidase conjugated antiDNA antibody was added to the lysate in the microtiter plate and incubated at $4{ }^{\circ} \mathrm{C}$ overnight. Following three washes with $300 \mu \mathrm{l}$ of incubation buffer, the presence of cytosolic nucleosomes was measured by adding $100 \mu \mathrm{l}$ of the substrate, ABTS. This was incubated in the microtiter plates for 5-20 $\mathrm{min}$ at room temperature and then the color reaction was quantitated on a spectrophotometer at $405 \mathrm{~nm}$. Experiments were performed within the linear range of the assay. Attached cells from parallel plates were counted and absorbance was normalized to cell number. A background (no lysate) measurement was taken and subtracted for each experiment.

Phosphatidylserine asymmetry: Another commonly used marker for apoptosis is loss of membrane phospholipid asymmetry. Normally, phosphatidylserine (PS) is located in the inner leaflet but during apoptosis PS appears in the outer leaflet. Labeled annexin V binds to PS. Propidium iodide is used to eliminate cells that have been labeled with annexin $\mathrm{V}$ due to membrane rupture rather than loss of membrane phospholipid assymetry. Equal cell numbers were plated and the next day switched to DM for $12 \mathrm{~h}$. Cells were trypsinized, pelleted and washed twice in phosphate-buffered saline (PBS). Samples were taken to the Cleveland Clinic Foundation Flow Cytometry Core for labeling and analysis. Cells were labeled with fluorscein-conjugated annexin $\mathrm{V}$ using an Apoptosis Detection Kit from R\&D Systems per manufacturer's instructions. Briefly, washed cells were resuspended in binding buffer at a concentration of $1 \times 10^{6} \mathrm{cells} / \mathrm{ml}$. To $100 \mu \mathrm{l}$ of this, $10 \mu \mathrm{l}$ of fluorscein-conjugated annexin $\mathrm{V}$ and $10 \mu \mathrm{l}$ of propidium iodide reagent were added. After $15 \mathrm{~min}$ at room temperature, $400 \mu \mathrm{l}$ of binding buffer was added and samples were analyzed immediately.

\section{Caspase 3 assays}

Caspase 3 colorimetric assays were performed using the Caspase 3 Colorimetric Assay Kit from R\&D Systems per manufacturer's instructions. Cells were plated and the next day switched to fresh GM or DM for various times prior to being rinsed in PBS. Attached cells were lysed in $50 \mu \mathrm{l}$ of lysis buffer. Following sedimentation, $50 \mu \mathrm{l}$ of lysate was incubated with $50 \mu \mathrm{l}$ of $2 \times$ reaction buffer supplemented with $0.5 \mu \mathrm{l} \mathrm{DTT}$ and $5 \mu \mathrm{l}$ of the caspase 3 colorimetric substrate, DEVD-pNA. Following $1-2 \mathrm{~h}$ of incubation at $37^{\circ} \mathrm{C}$, caspase-3 protease activity was measured on a spectrophotometer at $405 \mathrm{~nm}$. Experiments were performed within the linear range of the assay and absorbance was normalized by the protein concentration of each lysate as determined using Coomassie Protein Assay Reagent from Pierce. Fold increase in caspase 3 activity in DM was calculated relative to the absorbance value obtained from the lysate of cells incubated in fresh GM for $1 \mathrm{~h}$.

\section{Acknowledgements}

This work was partially supported by $\mathrm{NIH} / \mathrm{NCl}$ grant R01CA84212-01 awarded to CM Weyman and by start-up funds provided by Cleveland State University and the Cleveland Clinic Foundation.

\section{References}

1. Ellis RE, Yuan JY and Horvitz HR (1991) Mechanisms and functions of celldeath. Annu. Rev. Cell Biol. 7: 663-698

2. Raff MC (1992) Social controls on cell-survival and cell-death. Nature 356: $397-$ 400

3. Thompson CB (1995) Apoptosis in the pathogenesis and treatment of disease. Science 267: 1456-1462

4. Fidzianska A and Goebel HH (1991) Human ontogenesis 3. Cell death in fetal muscle. Acta Neuropathol 81: 572-577

5. Miller JB and Stockdale FE (1986) Developmental regulation of the multiple myogenic cell lineages of the avian embryo. J. Cell Biol. 103: 2197-2208

6. Sandri M, Cantini M, Massimino ML, Geromel V and Arslan P (1996) Myoblasts and myotubes in primary cultures deprived of growth factors undergo apoptosis. Basic Appl. Myol. 6: 257-260

7. Wang J and Walsh K (1996) Resistance to apoptosis conferred by Cdk inhibitors during myocyte differentiation. Science 273: 359-361

8. Tews DS and Goebel HH (1997) DNA-fragmentation and expression of apoptosis-related proteins in muscular dystrophies. Neuropathol. Appl. Neurobiol. 23: $331-338$

9. Tidball JG, Albrecht DE, Lokensgard BE and Spencer MJ (1995) Apoptosis precedes necrosis of dystrophin-deficient muscle. J. Cell Sci. 108: 2197-2204

10. Fidzianska A, Goebel HH and Warlo I (1990) Acute infantile spinal muscular atrophy. Muscle apoptosis as a proposed pathogenetic mechanism. Brain 113: 433- 445

11. Tews DS and Goebel HH (1996) DNA fragmentation and bcl-2 expression in infantile spinal muscular atrophy. Neuromusc. Disord. 6: 265-273

12. Skuk D and Tremblay JP (2000) Progress in myoblast transplantation: A potential treatment of dystrophies. Microsc. Res. Tech. 48: 213-222

13. Hartigan-O'Conner D and Chamberlain JS (2000) Developments in gene therapy for muscular dystrophy. Microsc. Res. Tech. 48: 223-238

14. Gussoni E, Blau HM and Kunkel LM (1997) The fate of individual myoblasts after transplantation into muscles of DMD patients. Nature Medicine 3: 970-977

15. Partridge T, Lu QL, Morris G and Hoffman E (1998) Is myoblast transplantation effective? Nature Med. 4: 1208-1209

16. Pagel CN and Morgan JE (1995) Myoblast transfer and gene therapy in muscular dystrophies. Microsc. Res. Tech. 30: 469-479

17. Perry RLS and Rudnicki MA (2000) Molecular mechanisms regulating myogenic determination and differentiation. Frontiers Biosci. 5: d750-767

18. Florini JR, Ewton DZ and Magri KA (1991) Hormones, growth factors, and myogenic differentiation. Annu. Rev. Physiol. 53: 201-216 
19. Florini JR, Magri KA, Ewton DZ, James $P$, Grindstaff $K$ and Rotwein $P$ (1991) "Spontaneous" differentiation of skeletal myoblasts is dependent upon autocrine secretion of insulin-like growth factor. J. Biol. Chem. 266: 1591715923

20. Coolican S, Samuel D, Ewton D, McWade F and Florini JR (1997) The mitogenic and myogenic actions of insulin-like growth factors utilize distinct signaling pathways. J. Biol. Chem. 272: 6653-6662

21. Jiang BH, Aoki M, Zheng JZ, Li J and Vogt PK (1999) Myogenic signaling of phosphatidylinositol 3-kinase requires the serine-threonine kinase Akt/protein kinase B. Proc. Natl. Acad. Sci. U.S.A. 96: 2077-2081

22. Weyman CM and Wolfman A (1998) Mitogen-activated protein kinase kinase (MEK) activity is required for inhibition of skeletal muscle differentiation by insulin-like growth factor-1 or fibroblast growth factor-2. Endocrinology 139 : $1794-1800$

23. Fujio Y, Guo K, Mano T, Mitsuuchi Y, Testa JR and Walsh K (1999) Cell cycle withdrawal promotes myogenic induction of Akt, a positive modulator of myocyte survival. Mol. Cell. Biol. 19: 5073-5082

24. Lawlor MA and Rotwein $P$ (2000) Insulin-like growth factor-mediated cell survival: central roles for Akt and cyclin-dependent kinase inhibitor p21. Mol. Cell. Biol. 20: 8983-8995

25. Salvesen G and Dixit VM (1997) Caspases: Intracellular signaling by proteolysis. Cell 91: 443-446

26. Thornberry N and Lazebnik Y (1998) Caspases: Enemies within. Science 281 $1312-1316$

27. Rodriguez J and Lazebnik $Y$ (1999) Caspase-9 and APAF-1 form an active holoenzyme. Genes Dev. 13: 3179-3184

28. Green DR (1998) Apoptotic pathways: The roads to ruin. Cell 94: 695-698

29. Weyman CM, Ramocki MB, Taparowsky EJ and Wolfman A (1997) Distinct signaling pathways regulate transformation and inhibition of skeletal muscle differentiation by oncogenic Ras. Oncogene 14: 697-704

30. Konieczny SF, Drobes BL, Menke SL and Taparowsky EJ (1989) Inhibition of myogenic differentiation by the $\mathrm{H}$-ras oncogene is associated with the downregulation of the MyoD1 gene. Oncogene 4: 473-481
31. Enkemann SA, Konieczny SF and Taparowsky EJ (1990) Adenovirus 5 E1A represses muscle-specific enhancers and inhibits expression of the myogenic regulatory factor genes, MyoD1 and myogenin. Cell Growth Differ. 1: 375-382

32. Cardone MH, Roy N, Stennicke HR, Salvesen GS, Franke TF, Stanbridge E, Frisch S and Reed JC (1998) Regulation of cell death protease caspase 9 by phosphorylation. Science 282: 1318-1321

33. Bonni A, Brunet A, West A, Sandeep RA, Takasu MA and Greenberg ME (1999) Cell survival promoted by the Ras-MAPK signaling pathway by transcriptiondependent and -independent mechanisms. Science 286: 1358-1362

34. Brown TL, Patil S, Cianci CD, Morrow JS and Howe PH (1999) Transforming growth factor beta induces caspase 3-independent cleavage of alphall-spectrin (alpha-fodrin) coincident with apoptosis. J. Biol. Chem. 274: 23256-23262

35. Miyashita T, Nagao K, Krajewski S, Salvesen G, Reed JC, Inoue T and Yamada M (1998) Investigation of glucocorticoid-induced apoptotic pathway:processing of caspase- 6 but not caspase-3. Cell Death Differ. 5: 1034-1041

36. Moisset PA, Bonham L, Skuk D, Koeberl D, Brusse V, Goulet M, Roy B, Asselin I, Miller AD and Tremblay JP (2000) Systemic production of human granulocyte colony stimulating factor in nonhuman primates by transplantation of genetically modified myoblasts. Hum. Gene Ther. 11: 1277-1288

37. Olson E (1992) Interplay between proliferation and differentiation. Dev. Biol. 154: $261-272$

38. Weyman CM and Wolfman A (1997) Oncogenic Ras-induced secretion of a novel inhibitor of skeletal myoblast differentiation. Oncogene 15: 2521-2528

39. Campbell SL, Khosravi-Far R, Rossman KL, Clark GJ and Der CJ (1998) Increasing complexity of Ras signaling. Oncogene 17: 1395-1413

40. Ramocki MB, Johnson SE, White MA, Ashendel CL, Koniescny SF and Taparowsky EJ (1997) Signaling through mitogen-activated protein kinase and Rac/Rho does not duplicate the effects of activated Ras on skeletal myogenesis. Mol. Cell. Biol. 17: 3547-3555 